\title{
Direct observation of spin-polarized bulk bands in an inversion-symmetric semiconductor
}

\author{
J. M. Riley ${ }^{1}$, F. Mazzola ${ }^{2}$, M. Dendzik ${ }^{3}$, M. Michiardi ${ }^{3}$, T. Takayamaa, ${ }^{4}$, L. Bawden ${ }^{1}$, C. Granerød ${ }^{2}$, \\ M. Leandersson ${ }^{6}$, T. Balasubramanian ${ }^{6}$, M. Hoesch ${ }^{7}$, T. K. Kim ${ }^{7}$, H. Takagi ${ }^{4,5}$, W. Meevasana ${ }^{8,9}$, \\ Ph. Hofmann ${ }^{3}$, M. S. Bahramy ${ }^{10,11}$, J. W. Wells ${ }^{2}$ and P. D. C. King ${ }^{1 \star}$
}

\begin{abstract}
Methods to generate spin-polarized electronic states in nonmagnetic solids are strongly desired to enable all-electrical manipulation of electron spins for new quantum devices ${ }^{1}$. This is generally accepted to require breaking global structural inversion symmetry ${ }^{1-5}$. In contrast, here we report the observation from spin- and angle-resolved photoemission spectroscopy of spin-polarized bulk states in the centrosymmetric transition-metal dichalcogenide $\mathrm{WSe}_{2}$. Mediated by a lack of inversion symmetry in constituent structural units of the bulk crystal where the electronic states are localized ${ }^{6}$, we show how spin splittings up to $\sim 0.5 \mathrm{eV}$ result, with a spin texture that is strongly modulated in both real and momentum space. Through this, our study provides direct experimental evidence for a putative locking of the spin with the layer and valley pseudospins in transition-metal dichalcogenides ${ }^{7,8}$, of key importance for using these compounds in proposed valleytronic devices.
\end{abstract}

The powerful combination of inversion symmetry $[E(\mathbf{k}, \uparrow)=E(-\mathbf{k}, \uparrow)]$ with time-reversal symmetry $[E(\mathbf{k}, \uparrow)=E(-\mathbf{k}, \downarrow)]$ ensures that electronic states of non-magnetic centrosymmetric materials must be doubly spin-degenerate. If inversion symmetry is broken, however, relativistic spin-orbit interactions can induce a momentum-dependent spin splitting via an effective magnetic field imposed by spatially-varying potentials. If the resulting spin polarizations can be controllably created and manipulated, they hold enormous promise to enable a range of new quantum technologies. These include routes towards electrical control of spin precession for spin-based electronics ${ }^{1,9}$, new ways to engineer topological states ${ }^{10,11}$ and possible hosts of Majorana fermions for use in quantum computation ${ }^{5}$. There are two generally accepted methods for stabilizing spin-polarized states without magnetism, both exploiting breaking of global inversion symmetry. Structural inversion asymmetry can be created in a centrosymmetric host by imposing an electrostatic potential gradient, for example within an asymmetric quantum well, leading to Rashba-split ${ }^{12}$ states localized at surfaces or interfaces ${ }^{13-16}$. Alternatively, a lack of global inversion symmetry in the unit cell can mediate spin splitting of the bulk electronic states, either through a Dresselhaus-type interaction ${ }^{17}$, or a recently discovered bulk form of the Rashba effect ${ }^{4,18}$.
Here, we present direct experimental evidence that $2 \mathrm{H}$-WSe $\mathrm{W}_{2}$, a material which retains bulk inversion symmetry, nonetheless exhibits a large spin polarization of its bulk electronic states. This layered compound is composed of stacked Se-W-Se planes (Fig. 1a), each of which contains an in-plane net dipole moment which is proposed to lead to a strong spin-valley coupling for an isolated monolayer ${ }^{19-21}$. The bulk unit cell contains two such monolayers, stacked in a staggered 'AB' configuration, restoring inversion symmetry and necessitating spin degeneracy of the bulk electronic states. Nevertheless, combining spin- and angle-resolved photoemission spectroscopy (ARPES) with electronic structure calculations, we observe a large layer- and momentum-dependent spin polarization of these bulk bands.

We first summarize the bulk electronic structure of $\mathrm{WSe}_{2}$ (Fig. 1). The material is known to be a semiconductor, consistent with our experimental observations, where we find the Fermi level located within the bandgap. We find the band extrema of the valence bands at $\Gamma$ and $\mathrm{K}$ to be almost degenerate ${ }^{22}$, but here can resolve that the valence band maximum is located at the bulk $\Gamma$ point, with significant dispersion of these zone-centre states along the surface normal $\left(k_{\mathrm{z}}\right)$ direction (Fig. 1c,e). Our measured band dispersions are in excellent agreement with those calculated from density functional theory (DFT; see also Supplementary Fig. 1), confirming that we are probing the bulk electronic states of $\mathrm{WSe}_{2}$. The broad total bandwidth of more than $4 \mathrm{eV}$ of the cosine-like upper valence bands along $\Gamma-\mathrm{A}$ reflects the spatially extended nature of $\mathrm{W} 5 d$ and Se $5 p$ orbitals from which these states predominantly derive.

As well as these dispersive states we find a series of quasitwo-dimensional states, predominantly of planar $d_{x^{2}-y^{2}}, d_{x y}$ and $p_{x / y}$ orbital character. The small overlap of these orbitals along the $z$ direction, combined with suppressed interlayer hopping due to spin-orbit coupling ${ }^{7}$, results in minimal dispersion along $k_{z}$, while their extended nature in-plane ensures significant dispersion throughout the surface Brillouin zone (Fig. 1d). The lowest binding energy two-dimensional states form a pair of hole-like bands centred at the Brillouin zone corners, contributing concentric, approximately circular pockets near the band top. These become trigonally warped as they grow in size with increasing binding energy, eventually merging with the zone-centre bands to form

\footnotetext{
${ }^{1}$ SUPA, School of Physics and Astronomy, University of St Andrews, St Andrews, Fife KY16 9SS, UK, ${ }^{2}$ Department of Physics, Norwegian University of Science and Technology (NTNU), N-7491 Trondheim, Norway, ${ }^{3}$ Department of Physics and Astronomy, Interdisciplinary Nanoscience Center (iNANO), Aarhus University, 8000 Aarhus C, Denmark, ${ }^{4}$ Department of Physics, University of Tokyo, Hongo, Tokyo 113-0033, Japan, ${ }^{5}$ Max Planck Institute for Solid State Research, 70569 Stuttgart, Germany, ${ }^{6}$ MAX IV Laboratory, Lund University, P. O. Box 118, 22100 Lund, Sweden, 7 Diamond Light Source, Harwell

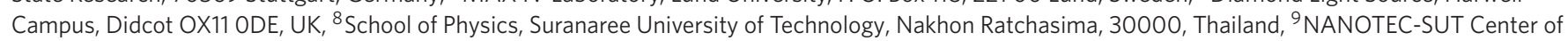
Excellence on Advanced Functional Nanomaterials, Suranaree University of Technology, Nakhon Ratchasima 30000 , Thailand, ${ }^{10}$ Quantum-Phase Electronics Center and Department of Applied Physics, The University of Tokyo, Tokyo 113-8656, Japan, ${ }^{11}$ RIKEN center for Emergent Matter Science (CEMS), Wako 351-0198, Japan. *e-mail: philip.king@st-andrews.ac.uk
} 
a

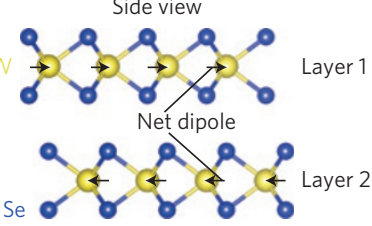

Top view

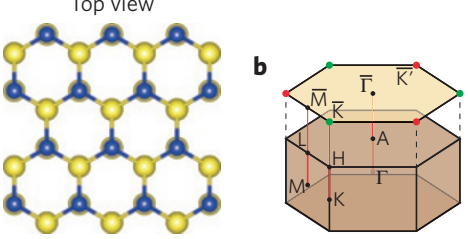

c

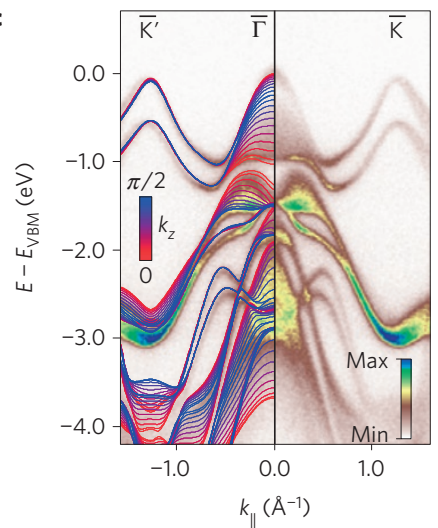

d
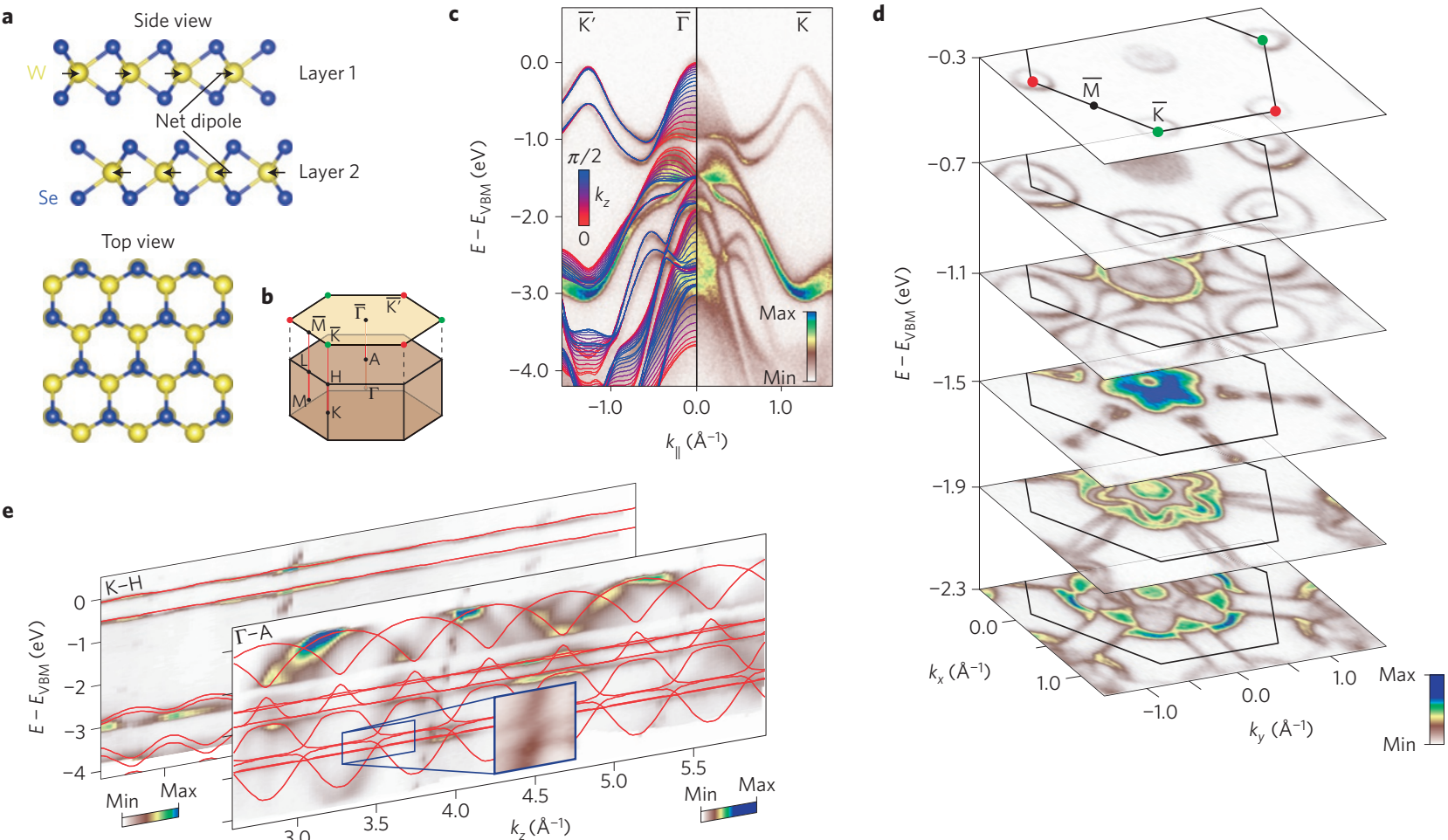

$k_{y}\left(\AA^{-1}\right.$

Min

Figure 1 | Bulk electronic structure of $\mathbf{W S e}_{2}$. $\mathbf{a}$, Side and top views of the bulk crystal structure of WSe $e_{2}$. The unit cell contains two Se-W-Se units in which there is a net in-plane dipole pointing to the right and left, respectively. $\mathbf{b}$, Corresponding bulk and surface Brillouin zone. c,d, ARPES measurements ( $h v=125 \mathrm{eV}, T=30 \mathrm{~K}$ ) of the electronic structure along the $\overline{\mathrm{K}^{\prime}}-\bar{\Gamma}-\overline{\mathrm{K}}$ direction (c) and isoenergy contours throughout the surface Brillouin zone (d) reveal

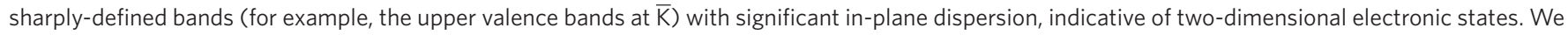
also observe broader 'filled-in' pockets of spectral weight characteristic of three-dimensional states, where the finite $k_{z}$ resolution of ARPES leads to broadening. e, We directly confirm this absence or presence, respectively, of significant $k_{z}$ dispersion from photon-energy-dependent ARPES measurements (Methods and Supplementary Fig. 1). Our measured electronic structure is in excellent agreement with that calculated from density functional theory (solid lines in $\mathbf{c}, \mathbf{e}$ ), confirming that we are probing the bulk electronic states of WSe.

bone-shaped pockets centred at $\overline{\mathrm{M}}$. The large splitting of $\sim 0.5 \mathrm{eV}$ of the top of these bands at $\overline{\mathrm{K}}$ signifies the strong atomic spinorbit interaction in this compound, which is further reflected by our observation of hybridization gaps, for example between twoand three-dimensional states along the $\Gamma$-A line (Fig. 1e). Despite such strong spin-orbit coupling, we stress that all states remain spindegenerate in our calculations, as expected from the bulk inversion symmetry of the crystal.

Intriguingly, however, our spin-resolved photoemission measurements reveal a strong spin polarization of the upper pair of valence band states at the $\overline{\mathrm{K}}$ point of the Brillouin zone (Fig. 2). The measured polarization is entirely out of the surface plane within experimental error, with up (down) orientation for the upper (lower) valence band, respectively. From fitting the measured energy distribution curves (EDCs, described in Methods), we estimate the magnitude of the spin polarization to exceed $90 \%$, suggestive of an almost fully spin-polarized band. Moreover, the signs of all polarizations are reversed at the $\overline{\mathrm{K}^{\prime}}=-\overline{\mathrm{K}}$ point, confirming that time-reversal symmetry remains unbroken, and thus there is no net magnetic moment. This indicates a nonmagnetic origin of the observed spin polarization, seemingly at odds with the centrosymmetric nature of the bulk crystal structure (Fig. 1a). We attribute this to the local inversion asymmetry of individual $\mathrm{WSe}_{2}$ layers, leading to spin-polarized states whose texture is strongly modulated in both real and momentum space despite the global inversion symmetry of the unit cell. For these quasi-two-dimensional bands around $\overline{\mathrm{K}}$, our calculations reveal that the electronic wavefunctions are almost completely localized on individual Se-W-Se layers of the bulk crystal. This is consistent with a spin-orbit-mediated suppression of interlayer hopping predicted at the $\overline{\mathrm{K}}$ point for bilayer $\mathrm{WSe}_{2}$, which was proposed to lead to a strong coupling of the real spin with the layer pseudospin ${ }^{7}$. Such spin-layer locking was subsequently attributed as the origin of characteristic circularly and linearly polarized photoluminescence from bilayer $\mathrm{WSe}_{2}$ (ref. 8).

As in the bilayer, with the electronic wavefunctions localized on a single Se-W-Se layer (half of the unit cell) of the bulk crystal around $\overline{\mathrm{K}}$, the $\mathrm{D}_{6 \mathrm{~h}}$ symmetry of the crystal is effectively reduced to $\mathrm{D}_{3 \mathrm{~h}}$, allowing a net dipole moment within the $a b$-plane (Fig. 1a). A recent theory has established the general grounds by which such a lack of inversion symmetry of the crystal site point group can lead to a macroscopic spin polarization, driven by the local nature of spin-orbit coupling ${ }^{6}$. Indeed, our calculated bulk wavefunctions projected onto either $\mathrm{WSe}_{2}$ layer of the unit cell are almost fully spin polarized for the topmost two valence bands at $\overline{\mathrm{K}}$ (Fig. $2 \mathrm{~g}$ ). The $180^{\circ}$ rotation of neighbouring layers in $\mathrm{AB}$-stacked $\mathrm{WSe}_{2}$, however, ensures that the sign of the spin polarization is opposite between adjacent layers (Fig. 2h). This leads to a strong spin-layer locking ${ }^{7,8}$, with an overall spin degeneracy of the bulk electronic structure as required for a centrosymmetric material. Photoemission, being extremely surface sensitive, can be expected to predominantly probe the top layer of this material. We thus attribute the strong measured spin polarization we observe here to be a direct observation of a layer-localized spin-polarization of bulk electronic states in $\mathrm{WSe}_{2}$.

This is further supported by our photon-energy-dependent measurements (Fig. 2i), which show how the measured photoelectron spin polarization at $\overline{\mathrm{K}}$ can be tuned nearly to zero. Our model calculations (see also Supplementary Fig. 3 and 
a

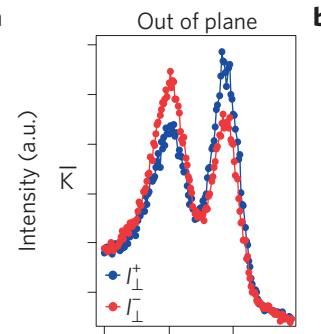

d

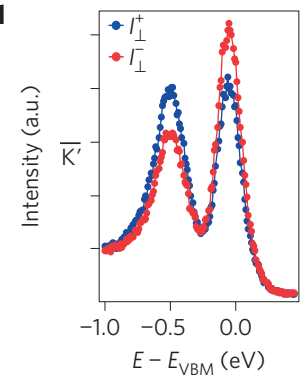

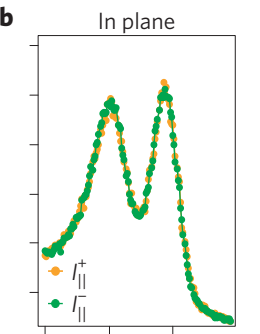

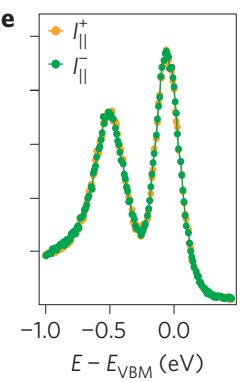

c
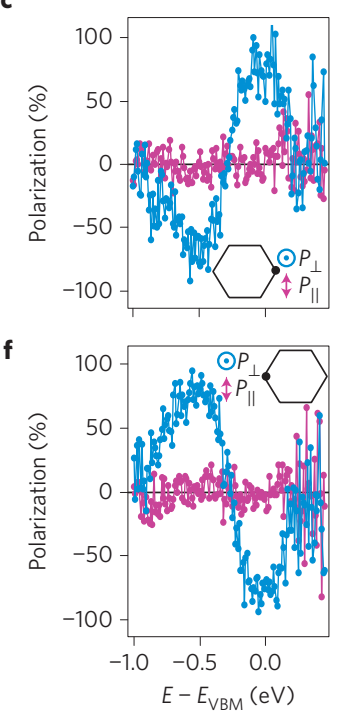

g

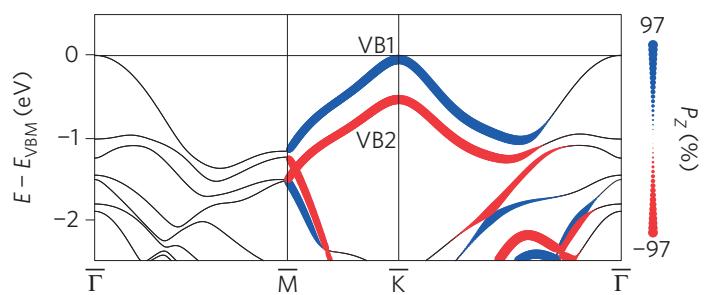

h

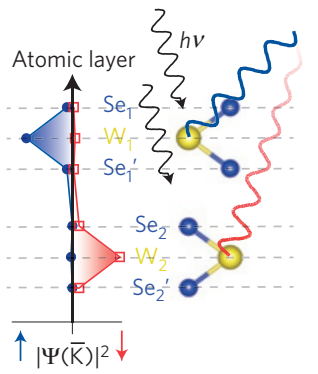

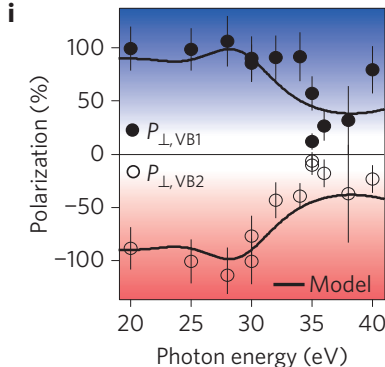

Figure 2 | Observation of spin-polarized bulk bands in an inversion-symmetric host. a,b, Energy distribution curves (in arbitrary units (a.u.)) from spin-resolved ARPES measurements $(h \nu=25 \mathrm{eV}, T=300 \mathrm{~K})$ at the $\overline{\mathrm{K}}$ point measured by the out-of-plane $\left(I_{\perp}\right)(\mathbf{a})$ and in-plane $\left(I_{\|}\right)(\mathbf{b})$ detectors in the Mott scattering chamber (corresponding measurements correcting for the finite Mott-detector efficiencies are shown in Supplementary Fig. 2). c, The extracted polarizations show a strong out-of-plane spin polarization, opposite for the two valence band peaks. $\mathbf{d}$-f, Plots as for $\mathbf{a}-\mathbf{c}$, but for the $\overline{K^{\prime}}$ point, showing that the signs of all components are reversed. $\mathbf{g}$, Our projection of the calculated bulk band structure onto the first layer of the unit cell reveals a strong spin polarization of electronic bands localized on this layer (for example, at $\bar{K}$ ), whose sign is reversed in the second layer of the unit cell, as shown in h. i, Measured spin polarization at $\bar{K}$ exhibiting a strong photon energy dependence. Error bars reflect an approximate estimate of the uncertainty in extracting the polarization from the experimental measurements, incorporating statistical errors in peak fitting, systematic errors and uncertainty in sample alignment. Our model calculations (solid line, see Supplementary Information) reveal how the photon energy-dependent modulations of the measured spin polarization result from interference between outgoing photoelectrons originating from the different layers of the crystal.
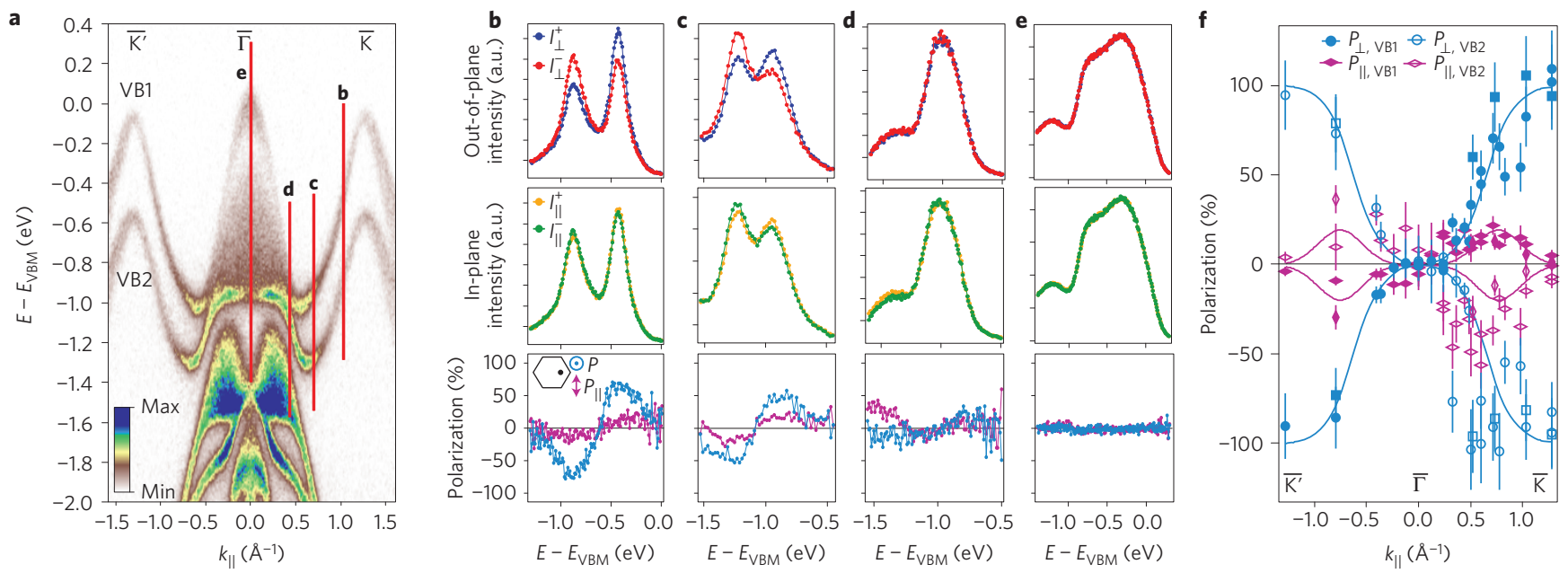

Figure 3 | Evolution of spin texture along $\overline{\mathbf{K}^{\prime}}-\bar{\Gamma}-\bar{K}$. a, Dispersion measured by ARPES ( $h v=125 \mathrm{eV}, T=30 \mathrm{~K}$ ) along the $\overline{\mathrm{K}^{\prime}}-\bar{\Gamma}-\overline{\mathrm{K}}$ direction. $\mathbf{b}-\mathbf{e}$, Energy distribution curves (EDCs; in arbitrary units (a.u.)) measured using spin-resolved photoemission ( $h v=25 \mathrm{eV}, T=300 \mathrm{~K}$ ) and corresponding extracted spin polarizations, measured at the locations labelled by the corresponding vertical lines in $\mathbf{a}$. $\mathbf{f}$, The out-of-plane $\left(P_{\perp}\right)$ and in-plane $\left(P_{\|}\right)$spin polarizations determined from fitting these and additional EDCs ( $h v=25 \mathrm{eV}, T=80 \mathrm{~K}$ (squares and vertical diamonds, respectively) and $T=300 \mathrm{~K}$ (circles and horizontal diamonds, respectively)) reveal some canting of the spin into the surface plane away from the $\bar{K}$ points, and a total suppression of the measured spin polarization around the zone centre. Error bars reflect an approximate estimate of the uncertainty in extracting the polarization from the experimental measurements, incorporating statistical errors in peak fitting, systematic errors and uncertainty in sample alignment. The lines in $\mathbf{f}$ are provided as guides to the eye.

associated discussion) show how this arises as a result of the interference $e^{23,24}$ of spin-up and spin-down polarized photoelectrons emitted from different layers of the material. This strongly supports our conclusions of a huge momentum-dependent spin splitting of up to $\sim 0.5 \mathrm{eV}$ for bulk states localized in a constituent layer of the unit cell in $\mathrm{WSe}_{2}$, with a spin orientation that is directly tied to the layer pseudospin. We note that the size of this observed spin splitting greatly exceeds spin-orbit-mediated splittings typically observed so far, even in surface Rashba systems with strong local in-plane field gradients $s^{14,25}$. This is because, here, the energy of the spin splitting at the band extrema is directly set by the atomic spin-orbit coupling strength ${ }^{7}$. 

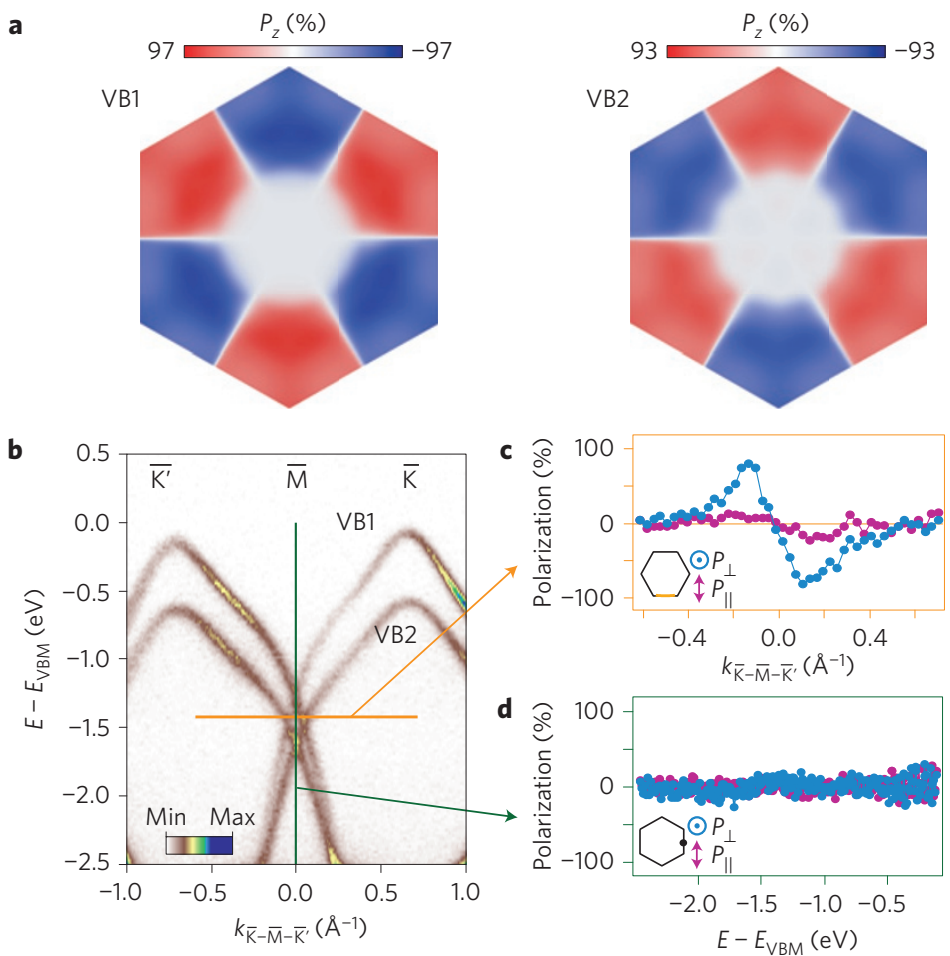

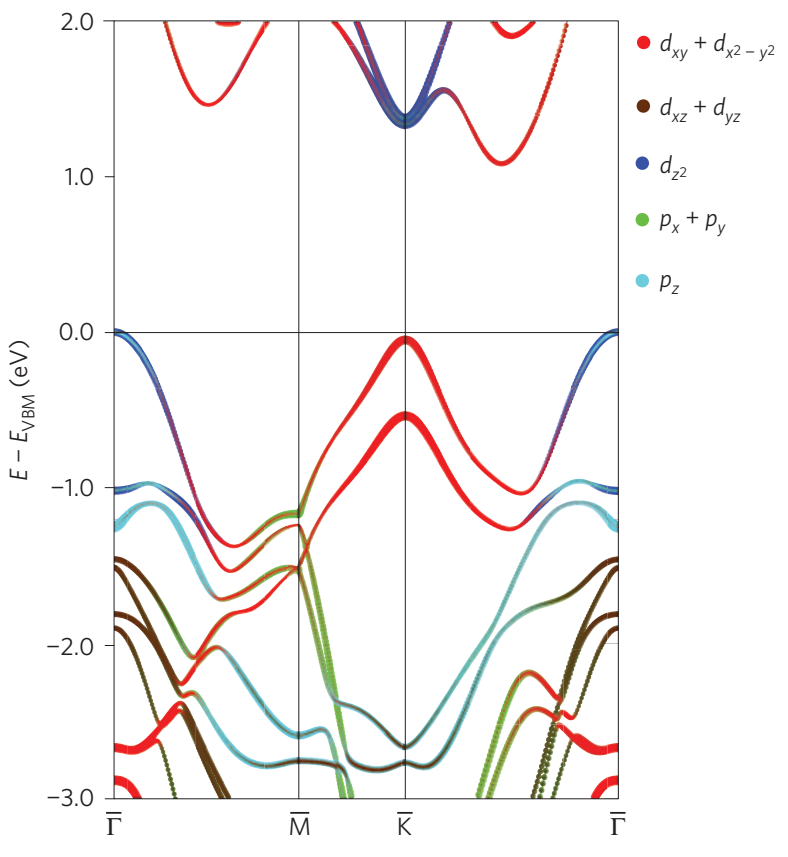

Figure 4 | Momentum-dependent suppression of layer-resolved spin polarization. a, Momentum dependence of the out-of-plane spin polarization of the top two valence bands throughout the Brillouin zone, calculated for the $k_{\mathrm{z}}=0$ plane and projected onto the first Se-W-Se layer of the unit cell. White regions indicate suppression of the layer-resolved spin-polarization. b-d, At the $\bar{M}$ point, our ARPES measurements $(h \nu=125 \mathrm{eV}, T=30 \mathrm{~K})(\mathbf{b})$ and corresponding spin polarization determined from spin-ARPES ( $h v=25 \mathrm{eV}, T=300 \mathrm{~K})$ along the coloured momentum (c) and energy (d) distribution curves show how this occurs through the crossing of strongly spin-polarized bands. e, In contrast, towards the zone centre, our orbitally projected band structure calculations reveal how this is correlated with the emergence of significant out-of-plane orbital character of the electronic states.

Figure 3 reveals how the underlying spin-polarized states evolve along the $\overline{\mathrm{K}}-\bar{\Gamma}-\overline{\mathrm{K}^{\prime}}$ direction. We find a marked suppression of the out-of-plane spin polarization approximately half way along this line, with negligible polarization observed around the zone centre. This is reproduced by our $a b$ initio calculations (Fig. 4a), and can be understood by considering the orbital character of the underlying states (Fig. 4e). Close to $\overline{\mathrm{K}}$, the electronic states are derived mostly from $d_{x y}$ and $d_{x^{2}-y^{2}}$ orbitals. There is thus significant orbital overlap within the surface plane, which, together with the net in-plane dipole, favours strong out-of-plane spin polarization ${ }^{26}$. Around $\bar{\Gamma}$, however, the orbital character becomes dominantly $d_{z^{2}} / p_{z}$-like, causing this component to be strongly suppressed, as found experimentally, while also driving the observed increase in dimensionality of the electronic states. Intriguingly, we also find a small in-plane spin component emerges along $\overline{\mathrm{K}^{\prime}}-\bar{\Gamma}-\overline{\mathrm{K}}$, which again switches sign either side of $\bar{\Gamma}$. This component would not naively be expected given the symmetry of the $\mathrm{Se}-\mathrm{W}$-Se layer, which has no net dipolar field along the out-of-plane direction, as we have confirmed by explicit slab calculations for an ideal bulk-like termination. The emergence of this component therefore reflects further complexity beyond that considered in our theoretical approach, such as small surface relaxations leading to a non-negligible contribution of the dipole out of the surface plane. We stress, however, that this has only a small effect and the predominant contributions to the strong outof-plane spin polarizations observed here are intrinsic to the bulk electric structure.

We also find a suppression of this out-of-plane spin polarization along the entire $\overline{\mathrm{M}}-\bar{\Gamma}$ direction. Unlike at the zone centre, however, this cannot be attributed to a change in orbital character: the electronic states close to $\overline{\mathrm{M}}$ are predominantly derived from planar orbitals, similar to around $\overline{\mathrm{K}}$, and we accordingly find strong layer-resolved spin polarizations of the underlying bands close to $\bar{M}$ (Fig. 4). Rather, the suppression of spin polarization along $\bar{M}-\bar{\Gamma}$ is mediated by the degeneracy of two oppositely polarized bands within a single layer. At the $\overline{\mathrm{M}}$ point itself, this is a natural consequence of time-reversal symmetry, as $\bar{M}$ is a time-reversal invariant momentum. Along the $\overline{\mathrm{M}}-\bar{\Gamma}$ line, such degeneracies are enforced by the combination of time-reversal with the rotational $\mathrm{D}_{3 \mathrm{~h}}$ symmetry of a single monolayer within the unit cell, ensuring that the out-of-plane component of the spin must have opposite sign in neighbouring sextants of the Brillouin zone.

Together, our calculations and experiment thus point to an extremely rich real- and momentum-space-dependent spin texture of bulk transition-metal dichalcogenides. They provide a direct demonstration of a pronounced coupling between the spin, valley and layer degrees of freedom, of key importance to widespread proposals to use these materials in exotic devices exploiting the valley pseudospin ${ }^{7,8,19-21,27-29}$. More generally, our experimental measurement of spin-polarized bulk electronic states in a centrosymmetric material opens a wealth of new opportunities for creating, probing and controlling spin and valley polarization in bulk solids via local inversion asymmetry.

\section{Methods}

ARPES. ARPES measurements were performed at the $\mathrm{I05}$ beamline of Diamond Light Source, UK, and spin-ARPES measurements at the I3 beamline of MAX-III synchrotron, Sweden ${ }^{30}$. Single-crystal samples of $\mathrm{WSe}_{2}$, grown by the chemical vapour transport method, were cleaved in situ and measured at temperatures ranging from 30 to $300 \mathrm{~K}$. Measurements were performed using $p$-polarized synchrotron light from 20 to $130 \mathrm{eV}$ (ARPES) and from 20 to $40 \mathrm{eV}$

(spin-ARPES), and employing Scienta R4000 hemispherical electron analysers. 
For the spin-ARPES measurements, a mini-Mott detector scheme was used, permitting simultaneous detection of the out-of-plane and one in-plane (along the analyser slit direction) component of the photoelectron $\operatorname{spin}^{30}$. A Sherman function of $S=0.17$ was used to generate the measured spin polarizations ${ }^{30}$

$$
P_{i}=\frac{\left(I_{i}^{+}-I_{i}^{-}\right)}{S\left(I_{i}^{+}+I_{i}^{-}\right)}
$$

where $P_{i}$ is the photoelectron spin polarization measured along the out-of-plane, $i=\perp$, or in-plane, $i=\|$, direction, and $I_{i}^{ \pm}$is the measured intensity on the individual detectors in the Mott scattering chamber, corrected by a relative detector efficiency calibration. To extract numerical values of the polarization, we fitted the measured EDCs to two Lorentzian peaks and a Shirley background, convolved with a Gaussian function to account for the instrumental resolution, with the corresponding Lorentzian peak areas used to derive the measured spin polarization. We also applied a geometrical correction to account for the finite angle between the sample and the electron spectrometer, and the corresponding influence of this on the spin polarization measured in the reference frame of the spectrometer. To determine the $k_{z}$ dispersion from photon-energy-dependent ARPES, we employed a free electron final state model

$$
k_{z}=\sqrt{2 m_{e} / \hbar^{2}}\left(V_{0}+E_{\mathrm{k}} \cos ^{2} \theta\right)^{1 / 2}
$$

where $\theta$ is the in-plane emission angle and $V_{0}$ is the inner potential. Our photon energy range covers more than six complete Brillouin zones along $k_{z}$, and we find best agreement taking an inner potential of $13 \mathrm{eV}$ and a $c$-axis lattice constant of $13.45 \AA$.

Calculations. Electronic structure calculations were performed within the context of density functional theory (DFT) using the modified Becke-Johnson exchange potential and Perdew-Burke-Ernzerhof correlation functional as implemented in the WIEN2K programme ${ }^{31}$. Relativistic effects, including spin-orbit coupling, were fully included. The Brillouin zone was sampled by a $12 \times 12 \times 6 k$-mesh. For the orbital and layer projection calculation, a tight binding Hamiltonian for the bulk band structure was constructed by downfolding the DFT results using maximally localized Wannier functions ${ }^{32-34}$, employing $\mathrm{W} 5 d$ and $5 s$ orbitals and Se $5 p$ and $5 s$ orbitals as a basis.

Received 1 June 2014; accepted 19 August 2014; published online 5 October 2014

\section{References}

1. Koo, H. C. et al. Control of spin precession in a spin-injected field effect transistor. Science 325, 1515-1518 (2009).

2. Hsieh, D. et al. A topological Dirac insulator in a quantum spin Hall phase. Nature 452, 970-974 (2008).

3. Bahramy, M. et al. Emergent quantum confinement at topological insulator surfaces. Nature Commun. 3, 1159 (2012).

4. Murakawa, H. et al. Detection of Berry's phase in a bulk Rashba semiconductor. Science 342, 1490-1493 (2013).

5. Mourik, V. et al. Signatures of Majorana fermions in hybrid superconductor-semiconductor nanowire devices. Science 336, 1003-1007 (2012).

6. Zhang, X., Liu, Q., Luo, J. W., Freeman, A. J. \& Zunger, A. Hidden spin polarization in inversion-symmetric bulk crystals. Nature Phys. 10, 387-393 (2014).

7. Gong, Z. et al. Magnetoelectric effects and valley-controlled spin quantum gates in transition metal dichalcogenide bilayers. Nature Commun. 4, 2053 (2013).

8. Jones, A. M. et al. Spin-layer locking effects in optical orientation of exciton spin in bilayer WSe $\mathrm{W}_{2}$. Nature Phys. 10, 130-134 (2014).

9. Datta, S. \& Das, B. Electronic analog of the electro-optic modulator. Appl. Phys. Lett. 56, 665-667 (1990).

10. Das, T. \& Balatsky, A. V. Engineering three-dimensional topological insulators in Rashba-type spin-orbit coupled heterostructures. Nature Commun. 4, 1972 (2013).

11. Zhou, J. J., Feng, W., Zhang, Y., Yang, S. A. \& Yao, Y. Engineering topological surface states and giant Rashba spin splitting in $\mathrm{BiTeI} / \mathrm{Bi}_{2} \mathrm{Te}_{3}$ heterostructures. Sci. Rep. 4, 3841 (2014).

12. Bychkov, Y. A. \& Rashba, E. I. Properties of a $2 \mathrm{D}$ electron gas with lifted spectral degeneracy. JETP Lett. 39, 78-81 (1984).
13. Nitta, J., Akazaki, T., Takayanagi, H. \& Enoki, T. Gate control of spin-orbit interaction in an inverted $\operatorname{In}_{0.53} \mathrm{Ga}_{0.47} \mathrm{As}_{\mathrm{In}} \mathrm{In}_{0.52} \mathrm{Al}_{0.48}$ As heterostructure. Phys. Rev. Lett. 78, 1335-1338 (1997).

14. Ast, C. R. et al. Giant spin splitting through surface alloying. Phys. Rev. Lett. 98, 186807 (2007).

15. King, P. D. C. et al. Large tunable Rashba spin splitting of a two-dimensional electron gas in $\mathrm{Bi}_{2} \mathrm{Se}_{3}$. Phys. Rev. Lett. 107, 096802 (2011).

16. King, P. D. C. et al. Quasiparticle dynamics and spin-orbital texture of the $\mathrm{SrTiO}_{3}$ two-dimensional electron gas. Nature Commun. 5, 3414 (2014).

17. Dresselhaus, G. Spin-orbit coupling effects in zinc blende structures. Phys. Rev. 100, 580-586 (1955).

18. Ishizaka, K. et al. Giant Rashba-type spin splitting in bulk BiTeI. Nature Mater. 10, 521-526 (2011).

19. Xiao, D., Liu, G-B., Feng, W., Xu, X. \& Yao, W. Coupled spin and valley physics in monolayers of $\mathrm{MoS}_{2}$ and other Group-VI dichalcogenides. Phys. Rev. Lett. 108, 196802 (2012).

20. Mak, K. F., He, K., Shan, J. \& Heinz, T. F. Control of valley polarization in monolayer $\mathrm{MoS}_{2}$ by optical helicity. Nature Nanotech. 7, 494-498 (2012).

21. Zeng, H., Dai, J., Yao, W., Xiao, D. \& Cui, X. Valley polarization in $\mathrm{MoS}_{2}$ monolayers by optical pumping. Nature Nanotech. 7, 490-493 (2012).

22. Finteis, T. et al. Occupied and unoccupied electronic band structure of $\mathrm{WSe}_{2}$. Phys. Rev. B 55, 10400-10411 (1997).

23. Zhu, Z-H. et al. Layer-by-layer entangled spin-orbital texture of the topological surface state in $\mathrm{Bi}_{2} \mathrm{Se}_{3}$. Phys. Rev. Lett. 110, 216401 (2013).

24. Zhu, Z-H. et al. Photoelectron spin-polarization control in the topological insulator $\mathrm{Bi}_{2} \mathrm{Se}_{3}$. Phys. Rev. Lett. 112, 076802 (2014).

25. Bihlmayer, G. et al. Enhanced Rashba spin-orbit splitting in $\mathrm{Bi} / \mathrm{Ag}(111)$ and $\mathrm{Pb} / \mathrm{Ag}(111)$ surface alloys from first principles. Phys. Rev. B 75, 195414 (2007).

26. Yuan, H. et al. Zeeman-type spin splitting controlled by an electric field. Nature Phys. 9, 563-569 (2013).

27. Jones, A. M. et al. Optical generation of excitonic valley coherence in monolayer WSe $\mathrm{W}_{2}$. Nature Nanotech. 8, 634-638 (2013).

28. Zhang, Y. J., Oka, T., Suzuki, R., Ye, J. T. \& Iwasa, Y. Electrically switchable chiral light-emitting transistor. Science 344, 725-728 (2014).

29. Xu, X., Yao, W., Xiao, D. \& Heinz, T. F. Spin and pseudospins in layered transition metal dichalcogenides. Nature Phys. 10, 343-350 (2014).

30. Berntsen, M. H. et al. A spin- and angle-resolving photoelectron spectrometer. Rev. Sci. Instrum. 81, 035104 (2010).

31. Blaha, P. et al. WIEN2K package, Version 10.1 (2010); http://www.wien2k.at

32. Souza, I. et al. Maximally localized Wannier functions for entangled energy bands. Phys. Rev. B 65, 035109 (2001).

33. Mostofi, A. A. et al. Wannier90: A tool for obtaining maximally localised Wannier functions. Comput. Phys. Commun. 178, 685-699 (2008).

34. Kuneš, J. et al. WIEN2WANNIER: From linearized augmented plane waves to maximally localized Wannier functions. Comput. Phys. Commun. 181, 1888-1895 (2010)

\section{Acknowledgements}

We gratefully acknowledge support from the Engineering and Physical Sciences Research Council, UK, the VILLUM foundation, the Calipso program, TRF-SUT Grant RSA5680052 and NANOTEC, Thailand through the CoE Network. P.D.C.K. acknowledges support from the Royal Society through a University Research Fellowship. M.S.B. was supported by a Grant-in-Aid for Scientific Research (S) (No. 24224009) from the Ministry of Education, Culture, Sports, Science and Technology (MEXT) of Japan. The experiments at the MAX IV Laboratory were made possible through funding from the Swedish Research Council and the Knut and Alice Wallenberg Foundation.

\section{Author contributions}

J.M.R., F.M., M.D., M.M., L.B., C.G., W.M., J.W.W. and P.D.C.K. measured the experimental data. J.M.R., F.M., J.W.W. and P.D.C.K. analysed the data. M.S.B. performed the electronic structure calculations. T.T. grew the samples. M.L. and T.B. maintained the spin-ARPES end stations and M.H. and T.K.K. maintained the ARPES end stations, respectively, and provided experimental support. P.D.C.K., J.W.W., M.S.B., P.H. and H.T. provided the project infrastructure. All authors discussed the results and their interpretation. P.D.C.K. and J.M.R. wrote the manuscript with input and discussion from all co-authors. P.D.C.K. was responsible for overall project planning and direction.

\section{Additional information}

Supplementary information is available in the online version of the paper. Reprints and permissions information is available online at www.nature.com/reprints. Correspondence and requests for materials should be addressed to P.D.C.K.

\section{Competing financial interests}

The authors declare no competing financial interests. 